\title{
The impact of rhizosphere soil structure and mucilage on root water uptake - a simulation study
}

\author{
MAGDALENA LANDL ${ }^{1 *}$, MAXIME PHALEMPIN ${ }^{2}$, DORIS \\ VETTERLEIN $^{2}$, STEFFEN SCHLÜTER ${ }^{2}$, MATHIEU

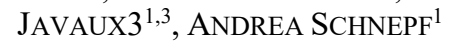

${ }^{1}$ Forschungszentrum Juelich GmbH, Agrosphere (IBG3), Juelich, Germany (*correspondence: m.landl@fzjuelich.de)

${ }^{2}$ Department of Soil Physics, Helmholtz Centre for Environmental Research -UFZ, Halle (Saale), Germany ${ }^{3}$ Earth and Life Institute/Environmental Sciences,

UCL, Louvain-la-Neuve, Belgium

The soil in the immediate vicinity of a root has structural and physical properties that differ from those of the bulk soil. A detailed understanding of how these differences influence the dynamics of root water uptake is still pending. In this work, we use a $1 \mathrm{D}$ radially symmetric rhizosphere model that is coupled to a $3 \mathrm{D}$ soil and root water transport model to investigate the impact of changes in rhizosphere soil structure and root mucilage exudation on root water uptake. In the rhizosphere model, we take into account gradual changes in soil hydraulic properties due to soil density gradients measured by X-ray microtomography and due to the presence of mucilage in the rhizosphere. The measured decrease in soil density in the immediate vicinity to the root increases water retention in the rhizosphere and therefore results in a delay of the water stress onset. The different drying/wetting rate of mucilage compared to the bulk soil leads to nonequilibrium relationships between water content and water potential resulting in less fluctuating water contents in the rhizosphere than in the bulk soil. Simulations are performed for different scenarios of micro-scale soil structure and mucilage concentrations. Our mechanistic simulation approach is an attempt to get a better understanding of the dynamics of root water uptake. 